\title{
Square Pixels to Hexagonal Pixel Structure Representation Technique
}

\author{
Barun kumar ${ }^{1}$, Pooja Gupta ${ }^{2}$ and Kuldip Pahwa ${ }^{3}$ \\ ${ }^{1} 4^{\text {th }}$ Semester M.Tech, Department of Electronics and Communication, MMEC MMU, \\ Mullana, Ambala, Haryana, India \\ ${ }^{2}$ Assistant Professor, Department of Electronics and Communication, MMEC MMU, \\ Mullana, Ambala, Haryana, India \\ ${ }^{3}$ Professor, Department of Electronics and Communication, MMEC MMU, Mullana \\ Ambala, Haryana, India \\ 1.barunku.ece@gmail.com, ${ }^{2}$ pooja.kaushik@mmumullana.org, \\ ${ }^{3}$ kuldip.pahwa@mmumullana.org
}

\begin{abstract}
The image processing is very important in several applications and have been using in them very efficiently. Normally all the pixels in every image are in shape of square grid but most of the time, the feature extraction from an image like image segmentation, image detection, edge detection, texture recognition etc becomes difficult to recognize in square pixel images. So one new approach called hexagonal pixel structure; has been designed to overcome the problems of square pixels structure. This paper gives an overview of different square pixels to hexagonal pixels representation techniques.
\end{abstract}

Keywords: Image processing, Hexagonal grid, Hexagonal addressing scheme

\section{Introduction}

Conventional acquisition devices acquire square pixel images but our approach is to manipulate square sampled image via software to produce hexagonal sample image, as there is no such hardware available today that can capture directly hexagonal sampled image. A lot of work has been carried out by the researcher over last 40 year on hexagonal image processing and the number of advantages of hexagonal structure over square structure has been observed and is as follows:

- Having a higher degree of circular symmetry

- Uniform fittings of pixels

- Reducing complex calculations in processing

- Enhancing image quality

- Consistent connectivity

- Having higher isotropy property

There are numbers of techniques available which can convert the square pixel to hexagonal pixel.

- Resampling method

- Pseudo hexagonal pixel

- Virtual hexagonal structure 
- Mimic hexagonal structure

- Spiral architecture

\section{Techniques to Convert Square Pixels to Hexagonal Pixels}

\subsection{Resampling Method}

In this technique, every alternate row is shifted to half the width of new pixels. This technique has angular resolution property which shows approximate $60^{\circ}$ angles with neighboring pixels. The central pixel shows equidistant property with its neighboring horizontal pixels and its value is equal to 1 but neighboring diagonal pixels do not represent equidistance property. Due to this reason this technique has a loss in image resolution.

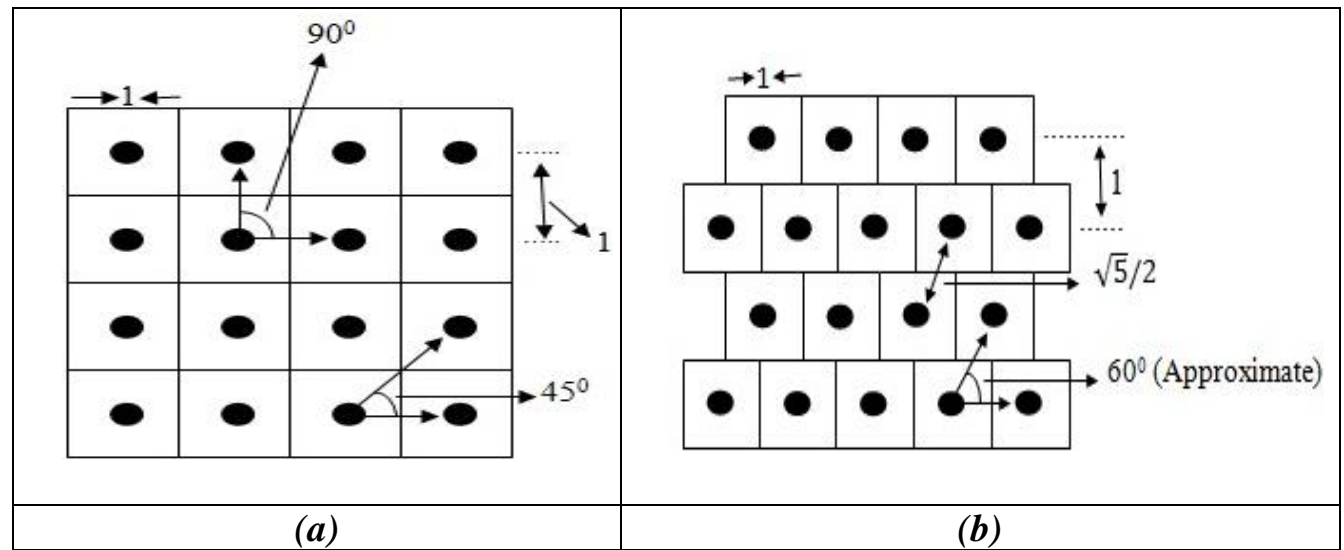

Figure 1. (a) Square Pixels on Hexagonal Sampling Grid (b) Hexagonal Structure Using Half Pixel Shift

\subsection{Pseudo Hexagonal Structure}

The pseudo hexagonal structure is designed from square pixels in the aspect ratio of 12:14 [13].

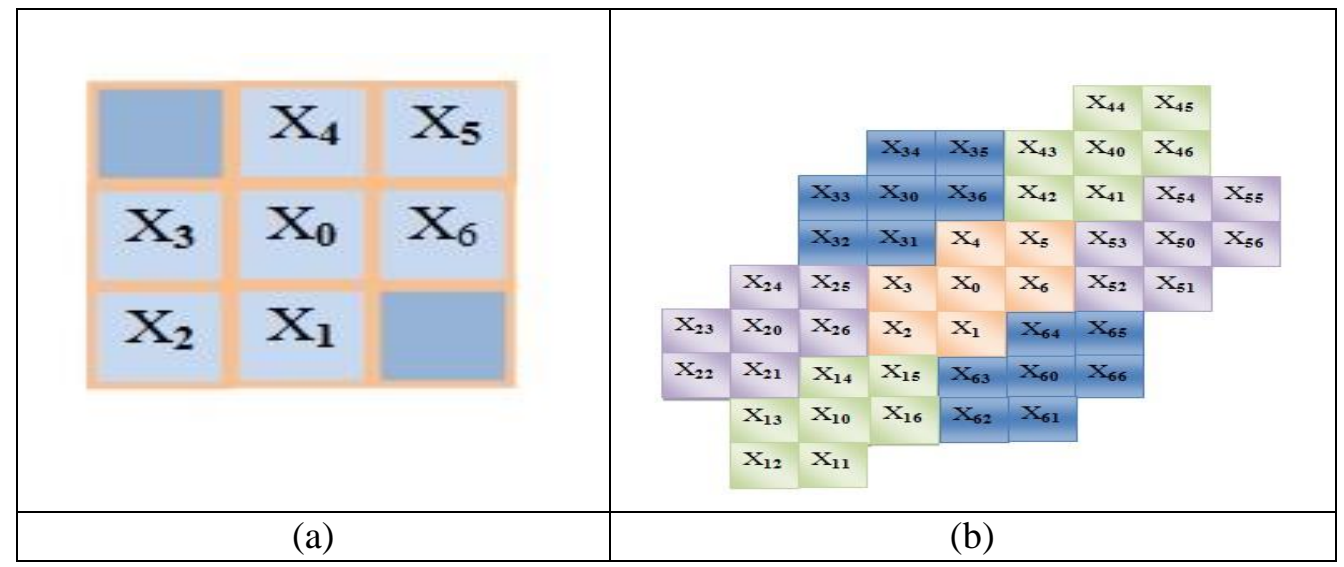

Figure 2. (a) Distribution of 7 Pixels Constructed with Labeled Rectangular Pixels (b) First 49 Pseudo Pixels Spiral Addresses 
The pseudo hexagonal structure is using only one rectangular pixel to represent a hexagonal pixel. The basic cluster of seven pixels with Spiral addresses 0 6 are represented in the above Figure 2. If the same scheme is repeated then any image on traditional structure can be covered and each of the pixels with a unique Spiral address can be labeled. In order to be consistent with the important property of hexagonal distribution that each such pixel has exactly six surrounding pixels, by considering six of the eight neighboring pixels for the centre pixels. The results of this hexagonal structure show poor screen resolution [14].

\subsection{Virtual Hexagonal Structure}

This technique is using a virtual spiral architecture in which spiral architecture is used during the processing part. The normal image in the traditional square grid is mapped into virtual spiral architecture and does the processing. Once the processing is done it is converted back into square grid and is displayed. It is different from all the above mimicking methods because it will neither create any distortion nor reduce resolution [13].

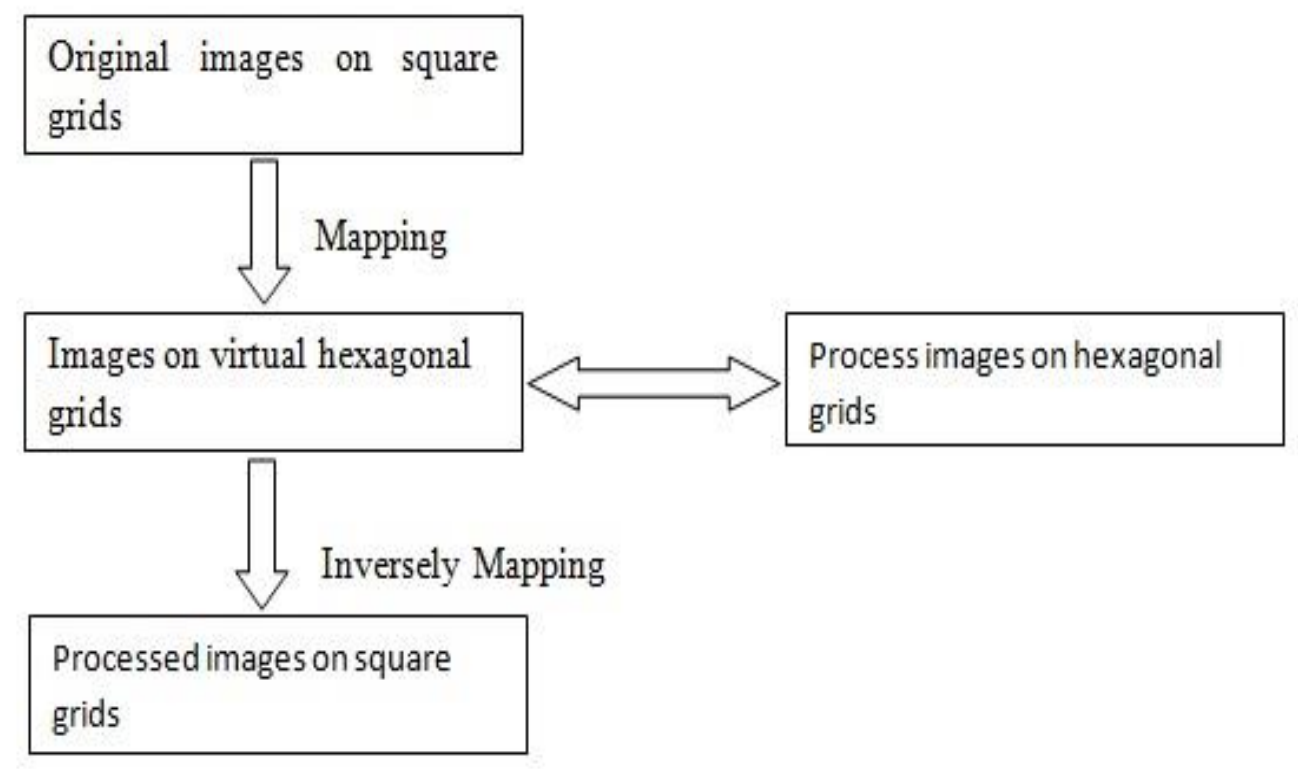

Figure 3. Image Processing on Virtual Spiral Architecture

\subsection{Mimic Hexagonal Structure}

In this technique, one hexagonal pixel means four square pixels and the equivalent grey level value is the average of these pixels. To construct the mimic hexagonal architecture, it starts with a collection of seven hexagonal pixels. These seven pixels are made by $28(4 \times 7)$ square pixels. A set of four square pixels which are adjacent to each other is used to mimic a hexagonal pixel. The seven mimic hexagonal pixels are numbered from 0 to 6 as shown in Fig.4. These numbers are also called Spiral Address of mimic hexagonal pixel. The grey label at each mimic hexagonal pixel is computed as the average of the grey value at the four hexagonal pixels, which together from the mimic hexagonal pixels. 


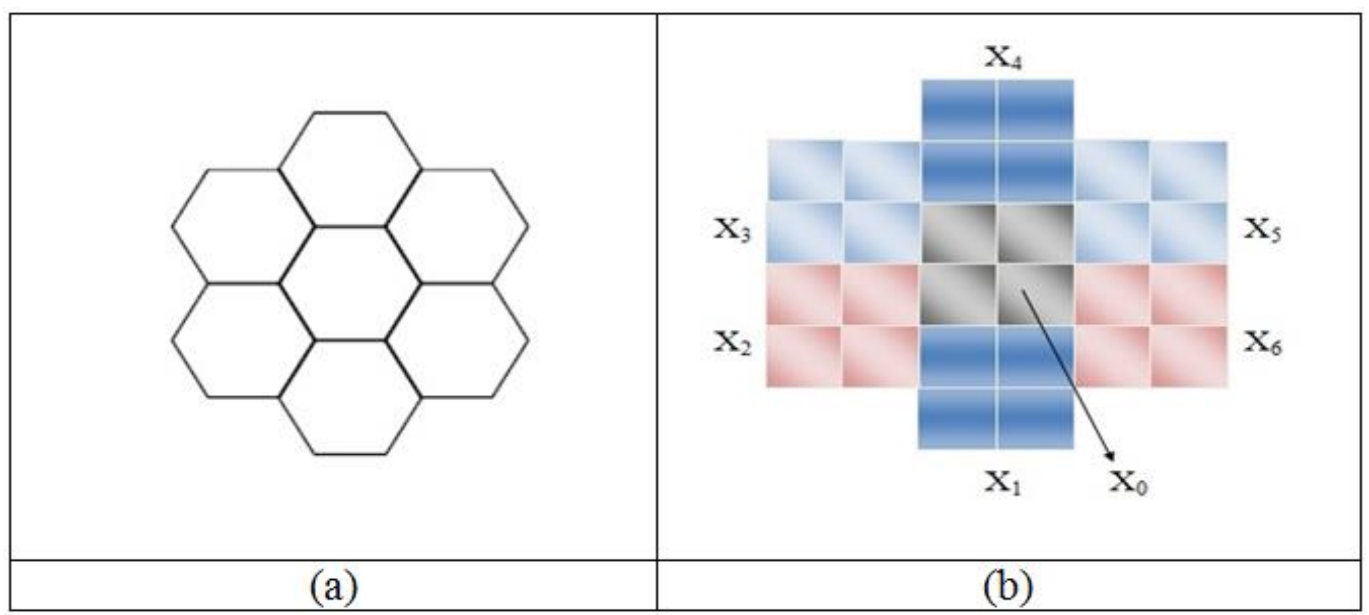

Figure 4. (a) A Cluster of 7 Hexagons (b) Distribution of Hexagonal Pixels Constructed from Square Pixels

\subsection{Spiral Structure Addressing}

To construct hexagonal pixels, each square pixel is first separated in $7 * 7$ smaller pixels called sub-pixels. Hexagonal pixels are designed using these 56 Sub-pixels and the structure is given in the following Figure 5.

\begin{tabular}{|c|c|c|c|c|c|c|c|c|}
\hline & & $\mathrm{x}$ & $\mathrm{x}$ & $\mathrm{x}$ & $\mathrm{x}$ & $\mathrm{x}$ & & \\
\hline & $\mathrm{x}$ & $\mathrm{x}$ & $\mathrm{x}$ & $\mathrm{x}$ & $\mathrm{x}$ & $\mathrm{x}$ & $\mathrm{x}$ & \\
\hline & $\mathrm{x}$ & $\mathrm{x}$ & $\mathrm{x}$ & $\mathrm{x}$ & $\mathrm{x}$ & $\mathrm{x}$ & $\mathrm{x}$ & \\
\hline $\mathrm{x}$ & $\mathrm{x}$ & $\mathrm{x}$ & $\mathrm{x}$ & $\mathrm{x}$ & $\mathrm{x}$ & $\mathrm{x}$ & $\mathrm{x}$ & $\mathrm{x}$ \\
\hline $\mathrm{x}$ & $\mathrm{x}$ & $\mathrm{x}$ & $\mathrm{x}$ & $\mathrm{x}$ & $\mathrm{x}$ & $\mathrm{x}$ & $\mathrm{x}$ & $\mathrm{x}$ \\
\hline & $\mathrm{x}$ & $\mathrm{x}$ & $\mathrm{x}$ & $\mathrm{x}$ & $\mathrm{x}$ & $\mathrm{x}$ & $\mathrm{x}$ & \\
\hline & $\mathrm{x}$ & $\mathrm{x}$ & $\mathrm{x}$ & $\mathrm{x}$ & $\mathrm{x}$ & $\mathrm{x}$ & $\mathrm{x}$ & \\
\hline & & $\mathrm{x}$ & $\mathrm{x}$ & $\mathrm{x}$ & $\mathrm{x}$ & $\mathrm{x}$ & & \\
\hline
\end{tabular}

Figure 5. The Structure of a Single Hexagonal Pixel

The size of each constructed hexagonal pixel is $12.5 \%$ bigger than each square pixel. Hence, the number of hexagonal pixels used are $12.5 \%$ lesser than the number of square pixels to cover the same image.

Total number of hexagonal pixels $=56$

Total number of square pixels $=49$

Thus, 
The size of each constructed hexagonal pixels is

[\{(total no of hexagonal sub-pixels) - (total no of square pixels) $\} /$ hexagonal sub-pixel $] \times$ 100

$$
=12.5 \%
$$

The design shown in Figure 5 implies the structure of one individual hexagonal pixel. A cluster of seven such hexagonal pixels can be found in the following Figure 6.

\begin{tabular}{|c|c|c|c|c|c|c|c|c|c|c|c|c|c|c|c|c|c|c|c|c|c|c|}
\hline & & & & & & & & & $\mathrm{x}_{4}$ & $x_{4}$ & $\mathrm{x}_{4}$ & $\mathrm{x}_{4}$ & $\mathrm{x}_{4}$ & & & & & & & & & \\
\hline & & & & & & & & $\mathrm{x}_{4}$ & $\mathrm{x}_{4}$ & $x_{4}$ & $\mathrm{x}_{4}$ & $\mathrm{x}_{4}$ & $\mathrm{x}_{4}$ & $\mathrm{x}_{4}$ & & & & & & & & \\
\hline & & & & & & & & $\mathrm{x}_{4}$ & $\mathrm{x}_{4}$ & $x_{4}$ & $\mathrm{x}_{4}$ & $\mathrm{x}_{4}$ & $\mathrm{x}_{4}$ & $\mathrm{x}_{4}$ & & & & & & & & \\
\hline & & & & & & & $\mathrm{x}_{4}$ & $\mathrm{x}_{4}$ & $\mathrm{x}_{4}$ & $x_{4}$ & $\mathrm{x}_{4}$ & $\mathrm{x}_{4}$ & $\mathrm{x}_{4}$ & $\mathrm{x}_{4}$ & $\mathrm{x}_{4}$ & & & & & & & \\
\hline & & $\mathrm{x}_{3}$ & $\mathrm{x}_{3}$ & $\mathrm{x}_{3}$ & $\mathrm{x}_{3}$ & $\mathrm{x}_{3}$ & $\mathrm{x}_{4}$ & $\mathrm{x}_{4}$ & $\mathrm{x}_{4}$ & $x_{4}$ & $\mathrm{x}_{4}$ & $\mathrm{x}_{4}$ & $\mathrm{x}_{4}$ & $\mathrm{x}_{4}$ & $\mathrm{x}_{4}$ & $x_{5}$ & $\mathrm{X}_{5}$ & $\mathrm{x}_{5}$ & $\mathrm{x}_{5}$ & $\mathrm{x}_{5}$ & & \\
\hline & $\mathrm{x}_{3}$ & $\mathrm{x}_{3}$ & $\mathrm{x}_{3}$ & $\mathrm{x}_{3}$ & $\mathrm{x}_{3}$ & $\mathrm{x}_{3}$ & $\mathrm{x}_{3}$ & $\mathrm{x}_{4}$ & $\mathrm{x}_{4}$ & $x_{4}$ & $\mathrm{x}_{4}$ & $\mathrm{x}_{4}$ & $\mathrm{x}_{4}$ & $\mathrm{x}_{4}$ & $\mathrm{x}_{5}$ & $x_{5}$ & $\mathrm{x}_{5}$ & $\mathrm{x}_{5}$ & $\mathrm{x}_{5}$ & $\mathrm{x}_{5}$ & $\mathrm{X}_{5}$ & \\
\hline & $\mathrm{x}_{3}$ & $\mathrm{x}_{3}$ & $\mathrm{x}_{3}$ & $\mathrm{x}_{3}$ & $\mathrm{x}_{3}$ & $\mathrm{x}_{3}$ & $\mathrm{x}_{3}$ & $\mathrm{x}_{4}$ & $x_{4}$ & $x_{4}$ & $\mathrm{x}_{4}$ & $\mathrm{x}_{4}$ & $\mathrm{x}_{4}$ & $\mathrm{x}_{4}$ & $\mathrm{X}_{5}$ & $\mathrm{x}_{5}$ & $\mathrm{x}_{5}$ & $\mathrm{X}_{5}$ & $\mathrm{x}_{5}$ & $\mathrm{x}_{5}$ & $\mathrm{x}_{5}$ & \\
\hline $\mathrm{x}_{3}$ & $\mathrm{x}_{3}$ & $\mathrm{x}_{3}$ & $\mathrm{x}_{3}$ & $\mathrm{x}_{3}$ & $\mathrm{x}_{3}$ & $\mathrm{x}_{3}$ & $\mathrm{x}_{3}$ & $\mathrm{x}_{3}$ & $x_{4}$ & $\mathrm{x}_{4}$ & $\mathrm{x}_{4}$ & $\mathrm{x}_{4}$ & $\mathrm{x}_{4}$ & $\mathrm{x}_{5}$ & $\mathrm{X}_{5}$ & $\mathrm{X}_{5}$ & $\mathrm{x}_{5}$ & $\mathrm{x}_{5}$ & $\mathrm{x}_{5}$ & $\mathrm{x}_{5}$ & $\mathrm{x}_{5}$ & $\mathrm{x}_{5}$ \\
\hline $\mathrm{x}_{3}$ & $\mathrm{x}_{3}$ & $\mathrm{x}_{3}$ & $\mathrm{x}_{3}$ & $\mathrm{x}_{3}$ & $\mathrm{x}_{3}$ & $\mathrm{x}_{3}$ & $\mathrm{x}_{3}$ & $\mathrm{x}_{3}$ & $\mathrm{x}_{0}$ & $\mathrm{x}_{0}$ & $\mathrm{x}_{0}$ & $\mathrm{x}_{0}$ & $\mathrm{x}_{0}$ & $\mathrm{x}_{5}$ & $\mathrm{x}_{5}$ & $x_{5}$ & $\mathrm{X}_{5}$ & $\mathrm{x}_{5}$ & $\mathrm{X}_{5}$ & $\mathrm{x}_{5}$ & $\mathrm{X}_{5}$ & $\mathrm{x}_{5}$ \\
\hline & $\mathrm{x}_{3}$ & $\mathrm{x}_{3}$ & $\mathrm{x}_{3}$ & $\mathrm{x}_{3}$ & $\mathrm{x}_{3}$ & $\mathrm{x}_{3}$ & $\mathrm{x}_{3}$ & $\mathrm{x}_{0}$ & $\mathrm{x}_{0}$ & $\mathrm{x}_{0}$ & $\mathrm{x}_{0}$ & $\mathrm{x}_{0}$ & $\mathrm{x}_{0}$ & $\mathrm{x}_{0}$ & $\mathrm{x}_{5}$ & $\mathrm{x}_{5}$ & $\mathrm{X}_{5}$ & $\mathrm{x}_{5}$ & $\mathrm{X}_{5}$ & $\mathrm{X}_{5}$ & $\mathrm{x}_{5}$ & \\
\hline & $\mathrm{x}_{3}$ & $\mathrm{x}_{3}$ & $\mathrm{x}_{3}$ & $\mathrm{x}_{3}$ & $\mathrm{x}_{3}$ & $\mathrm{x}_{3}$ & $\mathrm{x}_{3}$ & $\mathrm{x}_{0}$ & $\mathrm{x}_{0}$ & $\mathrm{x}_{0}$ & $\mathrm{x}_{0}$ & $\mathrm{x}_{0}$ & $\mathrm{x}_{0}$ & $\mathrm{x}_{0}$ & $\mathrm{X}_{5}$ & $\mathrm{x}_{5}$ & $\mathrm{x}_{5}$ & $\mathrm{X}_{5}$ & $\mathrm{x}_{5}$ & $\mathrm{X}_{5}$ & $\mathrm{X}_{5}$ & \\
\hline & & $\mathrm{x}_{3}$ & $\mathrm{x}_{3}$ & $\mathrm{x}_{3}$ & $\mathrm{x}_{3}$ & $\mathrm{x}_{3}$ & $\mathrm{x}_{0}$ & $\mathrm{x}_{0}$ & $\mathrm{x}_{0}$ & $\mathrm{x}_{0}$ & $\mathrm{x}_{0}$ & $\mathrm{x}_{0}$ & $\mathrm{x}_{0}$ & $\mathrm{x}_{0}$ & $\mathrm{x}_{0}$ & $\mathrm{x}_{5}$ & $\mathrm{x}_{5}$ & $\mathrm{X}_{5}$ & $\mathrm{X}_{5}$ & $\mathrm{x}_{5}$ & & \\
\hline & & $\mathrm{X}_{2}$ & $\mathrm{x}_{2}$ & $\mathrm{x}_{2}$ & $\mathrm{x}_{2}$ & $\mathrm{x}_{2}$ & $\mathrm{x}_{0}$ & $\mathrm{x}_{0}$ & $\mathrm{x}_{0}$ & $\mathrm{x}_{0}$ & $\mathrm{x}_{0}$ & $\mathrm{x}_{0}$ & $\mathrm{x}_{0}$ & $\mathrm{x}_{0}$ & $\mathrm{x}_{0}$ & $\mathrm{x}_{6}$ & $\mathrm{x}_{6}$ & $\mathrm{x}_{6}$ & $\mathrm{X}_{6}$ & $\mathrm{x}_{0}$ & & \\
\hline & $\mathrm{x}_{2}$ & $\mathrm{x}_{2}$ & $\mathrm{x}_{2}$ & $\mathrm{X}_{2}$ & $\mathrm{x}_{2}$ & $\mathrm{x}_{2}$ & $\mathrm{X}_{2}$ & $\mathrm{x}_{0}$ & $\mathrm{x}_{0}$ & $\mathrm{x}_{0}$ & $\mathrm{x}_{0}$ & $\mathrm{x}_{0}$ & $\mathrm{x}_{0}$ & $\mathrm{x}_{0}$ & $\mathrm{x}_{6}$ & $x_{6}$ & $\mathrm{x}_{6}$ & $\mathrm{x}_{6}$ & $\mathrm{X}_{6}$ & $\mathrm{x}_{6}$ & $\mathrm{x}_{6}$ & \\
\hline & $\mathrm{x}_{2}$ & $\mathrm{x}_{2}$ & $\mathrm{x}_{2}$ & $\mathrm{x}_{2}$ & $\mathrm{x}_{2}$ & $\mathrm{x}_{2}$ & $\mathrm{x}_{2}$ & $\mathrm{x}_{0}$ & $\mathrm{x}_{0}$ & $\mathrm{x}_{0}$ & $\mathrm{x}_{0}$ & $\mathrm{x}_{0}$ & $\mathrm{x}_{0}$ & $\mathrm{x}_{0}$ & $\mathrm{x}_{6}$ & $\mathrm{x}_{6}$ & $x_{6}$ & $\mathrm{x}_{0}$ & $\mathrm{x}_{6}$ & $\mathrm{x}_{6}$ & $\mathrm{x}_{6}$ & \\
\hline $\mathrm{x}_{2}$ & $\mathrm{x}_{2}$ & $\mathrm{X}_{2}$ & $\mathrm{x}_{2}$ & $\mathrm{x}_{2}$ & $\mathrm{X}_{2}$ & $\mathrm{x}_{2}$ & $\mathrm{x}_{2}$ & $\mathrm{x}_{2}$ & $\mathrm{x}_{0}$ & $\mathrm{x}_{0}$ & $\mathrm{x}_{0}$ & $\mathrm{x}_{0}$ & $\mathrm{x}_{0}$ & $\mathrm{x}_{6}$ & $\mathrm{x}_{6}$ & $\mathrm{x}_{6}$ & $\mathrm{x}_{6}$ & $\mathrm{x}_{6}$ & $\mathrm{X}_{6}$ & $\mathrm{x}_{6}$ & $\mathrm{x}_{6}$ & $\mathrm{x}_{6}$ \\
\hline $\mathrm{x}_{2}$ & $\mathrm{x}_{2}$ & $\mathrm{x}_{2}$ & $\mathrm{x}_{2}$ & $\mathrm{x}_{2}$ & $\mathrm{x}_{2}$ & $\mathrm{x}_{2}$ & $\mathrm{x}_{2}$ & $\mathrm{X}_{2}$ & $\mathrm{x}_{1}$ & $\mathrm{x}_{1}$ & $\mathrm{x}_{1}$ & $\mathrm{x}_{1}$ & $\mathrm{x}_{1}$ & $\mathrm{x}_{0}$ & $\mathrm{x}_{0}$ & $\mathrm{x}_{6}$ & $\mathrm{x}_{6}$ & $\mathrm{x}_{6}$ & $\mathrm{x}_{6}$ & $\mathrm{x}_{6}$ & $\mathrm{x}_{6}$ & $\mathrm{x}_{6}$ \\
\hline & $\mathrm{x}_{2}$ & $\mathrm{x}_{2}$ & $\mathrm{x}_{2}$ & $\mathrm{x}_{2}$ & $\mathrm{x}_{2}$ & $\mathrm{x}_{2}$ & $\mathrm{X}_{2}$ & $\mathrm{x}_{1}$ & $\mathrm{x}_{1}$ & $\mathrm{x}_{1}$ & $\mathrm{x}_{1}$ & $\mathrm{x}_{1}$ & $\mathrm{x}_{1}$ & $\mathrm{x}_{1}$ & $\mathrm{x}_{6}$ & $\mathrm{x}_{6}$ & $\mathrm{x}_{6}$ & $\mathrm{x}_{6}$ & $x_{0}$ & $\mathrm{x}_{0}$ & $\mathrm{x}_{6}$ & \\
\hline & $\mathrm{x}_{2}$ & $\mathrm{x}_{2}$ & $\mathrm{x}_{2}$ & $\mathrm{x}_{2}$ & $\mathrm{x}_{2}$ & $\mathrm{x}_{2}$ & $\mathrm{x}_{2}$ & $\mathrm{x}_{1}$ & $\mathrm{x}_{1}$ & $\mathrm{x}_{1}$ & $\mathrm{x}_{1}$ & $\mathrm{x}_{1}$ & $\mathrm{x}_{1}$ & $\mathrm{x}_{1}$ & $\mathrm{x}_{6}$ & $\mathrm{x}_{6}$ & $\mathrm{x}_{6}$ & $\mathrm{X}_{6}$ & $\mathrm{X}_{6}$ & $\mathrm{x}_{0}$ & $\mathrm{x}_{6}$ & \\
\hline & & $\mathrm{x}_{2}$ & $\mathrm{x}_{2}$ & $\mathrm{x}_{2}$ & $\mathrm{x}_{2}$ & $\mathrm{x}_{2}$ & $\mathrm{x}_{1}$ & $\mathrm{x}_{1}$ & $\mathrm{x}_{1}$ & $\mathrm{x}_{1}$ & $\mathrm{x}_{1}$ & $\mathrm{x}_{1}$ & $\mathrm{x}_{1}$ & $\mathrm{x}_{1}$ & $\mathrm{x}_{1}$ & $\mathrm{x}_{6}$ & $\mathrm{x}_{6}$ & $\mathrm{X}_{6}$ & $\mathrm{X}_{6}$ & $\mathrm{x}_{6}$ & & \\
\hline & & & & & & & $\mathrm{x}_{1}$ & $\mathrm{x}_{1}$ & $\mathrm{x}_{1}$ & $\mathrm{x}_{1}$ & $\mathrm{x}_{1}$ & $\mathrm{x}_{1}$ & $\mathrm{x}_{1}$ & $\mathrm{x}_{\mathrm{I}}$ & $\mathrm{x}_{1}$ & & & & & & & \\
\hline & & & & & & & & $\mathrm{x}_{1}$ & $\mathrm{x}_{1}$ & $\mathrm{x}_{1}$ & $\mathrm{x}_{1}$ & $\mathrm{x}_{1}$ & $\mathrm{x}_{1}$ & $\mathrm{x}_{1}$ & & & & & & & & \\
\hline & & & & & & & & $\mathrm{x}_{1}$ & $\mathrm{x}_{1}$ & $\mathrm{x}_{1}$ & $\mathrm{x}_{1}$ & $\mathrm{x}_{1}$ & $\mathrm{x}_{1}$ & $\mathrm{x}_{1}$ & & & & & & & & \\
\hline & & & & & & & & & $\mathrm{x}_{1}$ & $x_{1}$ & $\mathrm{x}_{1}$ & $\mathrm{x}_{1}$ & $\mathrm{x}_{1}$ & & & & & & & & & \\
\hline
\end{tabular}

Figure 6. Cluster of Seven Hexagonal Pixels

The addressing of these hexagonal pixels is done like spiral architecture. The address of central pixel is 0 and its neighboring 6 pixels have address 1-6. The complete addressing of hexagonal pixels is done by using base seven addresses only. This means that after 6 the next address will be 10. Figure 6 shows the complete addressing of cluster of $7^{2}=49$ square pixels.

This hexagonal structure retains the property of equal distance as all the neighboring pixels are equidistant from their central pixel. And this type of construction hardly introduces image distortion. 


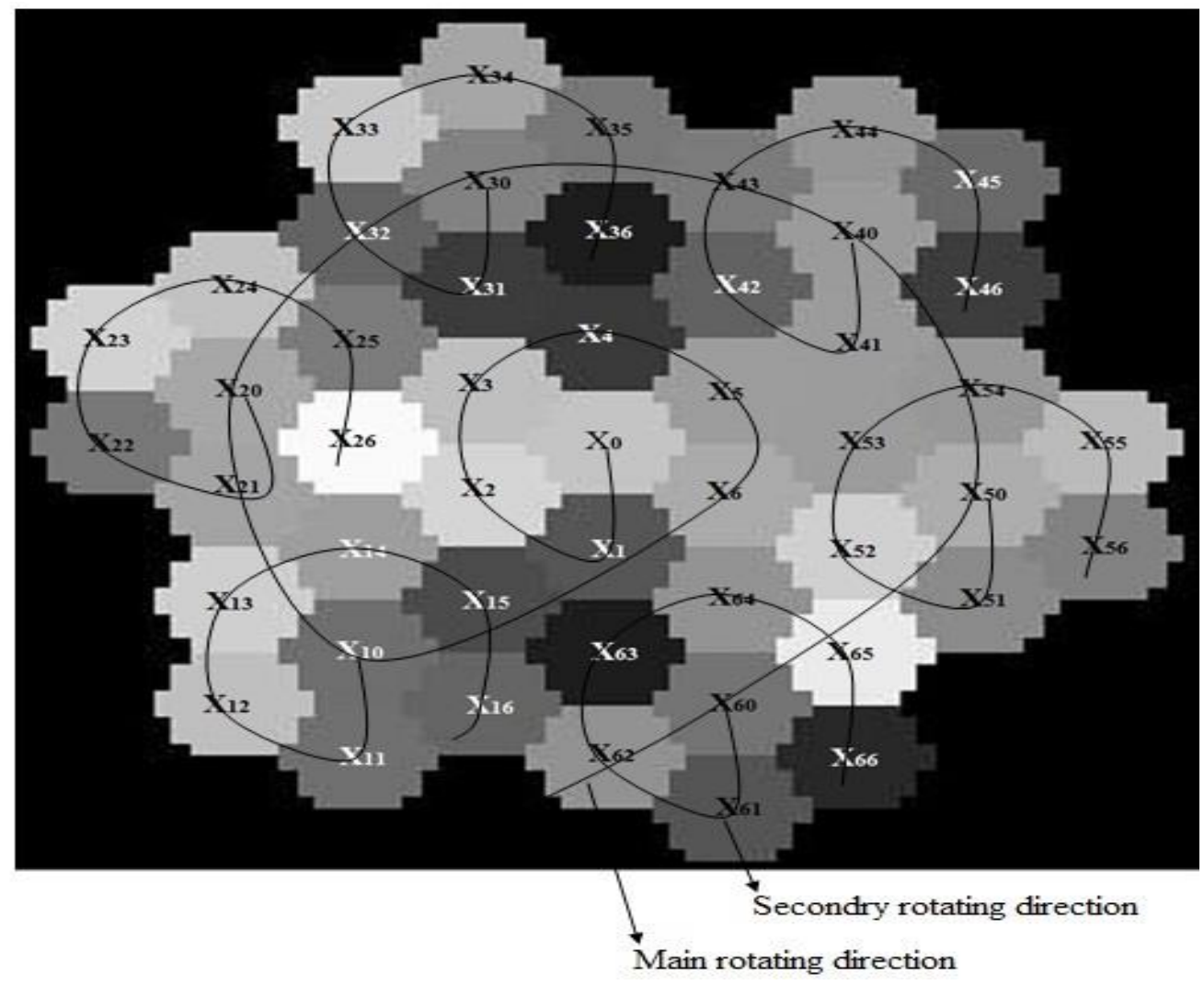

Figure 7. Spiral Architecture with Spiral Addressing

If $\mathrm{D}(\mathcal{X})$ is used to denote the location of the hexagonal pixel with spiral address architecture. Thus

$$
\mathrm{D}(0)=\left[\begin{array}{ll}
0 & 0
\end{array}\right]
$$

From Figure 6, it is easy to see that $\mathrm{D}(1)=[80], \mathrm{D}(2)=[4-7], \mathrm{D}(3)=[-4-7], \mathrm{D}(4)=[-8$ $0], \mathrm{D}(5)=[-47]$ and $\mathrm{D}(6)=[47]$

The shift for addresses 0 to 6 are base cases for the recursive algorithm. The algorithm for multiples of 10 is given by

$$
\begin{gathered}
D\left(x \times 10^{i}\right)=D\left(x \times 10^{i-1}\right)+2 D\left((x+1) \times 10^{i-1}\right) \\
D\left(6 \times 10^{i}\right)=D\left(6 \times 10^{i-1}\right)+2 D\left(10^{i-1}\right) \\
\text { For } i=1,2 \ldots, x=1,2 \ldots, 5 .
\end{gathered}
$$

While the location of the pixel with a given spiral address

Can be obtained by

$$
x_{n} x_{n-1} \ldots a_{1},\left(x_{i}=0,1,2 \ldots 6 . \text { For } i=1,2 \ldots, n .\right)
$$

$$
D\left(x_{n} x_{n-1} \ldots a_{1}\right)=\sum_{i=1}^{n} D\left(x_{i} \times 10^{i-l}\right)
$$

\section{Conclusion}

In this paper, a number of hexagonal conversion schemes have been discussed and it has been observed that spiral addressing scheme does not change image resolution and image distortion. It retains the advantages of real hexagonal system such as high degree of symmetry uniform connectivity, equidistant property and close pact form. The construction of this 
structure does not require complex computations and the location of each new hexagonal pixel can be calculated and obtained using this simple procedure. The following Figure 8 shows the construction of hexagonal pixel images using spiral addressing schemes for different pixel clusters.
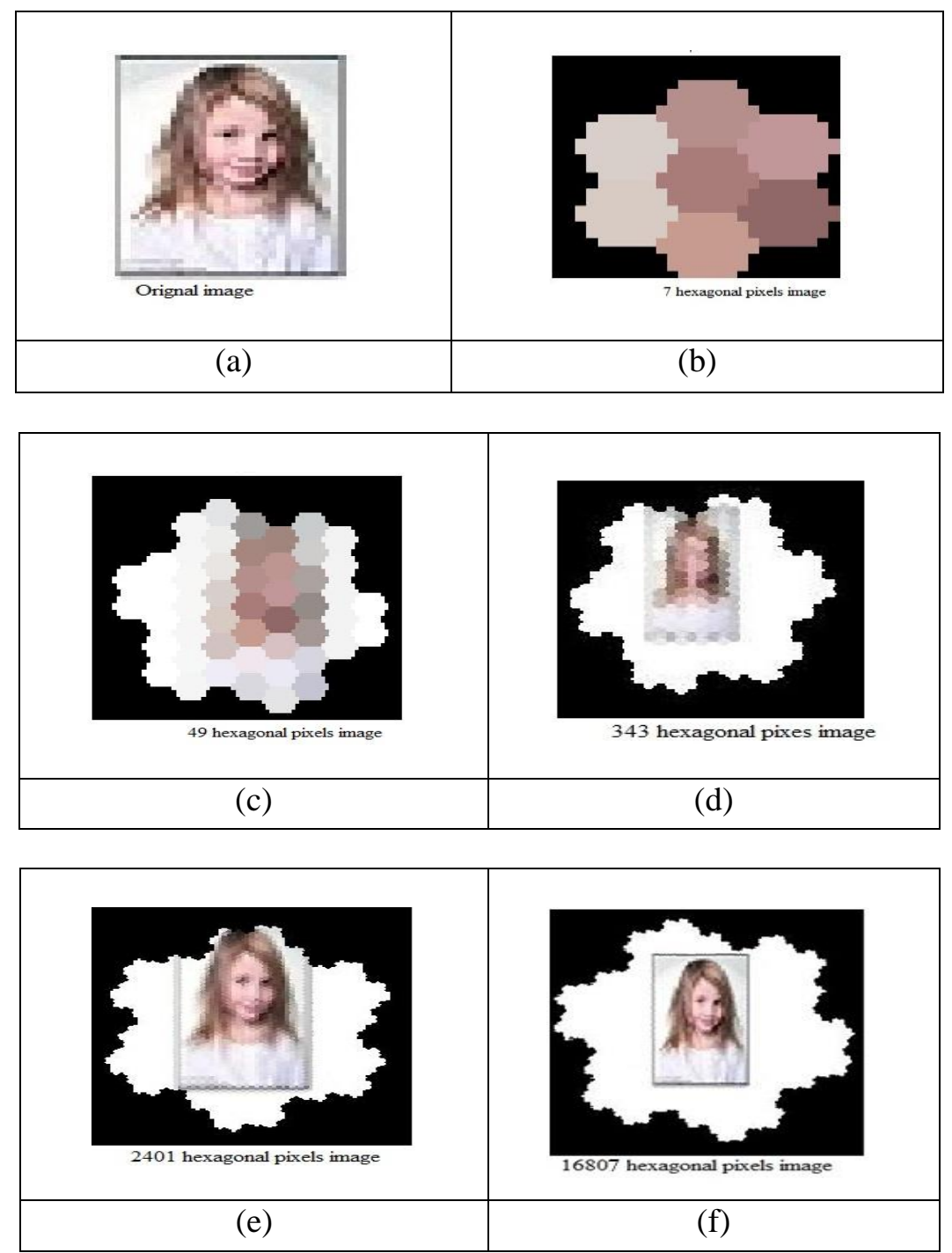

Figure 8. (a) Original Image of Square Pixels, (b) Cluster of 7 Hexagonal Pixels Image, (c) $7^{2}$ Hexagonal Pixels Image, (d) Cluster of $7^{3}$ Hexagonal Pixels Image, (e) Cluster of $7^{4}$ Hexagonal Pixels Image, (f) Cluster of $7^{5}$ Hexagonal Pixels Image

\section{References}

[1] H. Wang, M. Wang and T. Hintz, "VSA-based Fractal Image Compression", Journal of WSCG, (2005).

[2] M. B. Nourian and M. R. Aahmadzadeh, "Virtual Hexagonal Image Structure", Introduction of Hexagonal Image, 978-1-4673-6206-1/13 IEEE, (2013).

[3] R. C. Gonzales and R. E. Woods, "Digital Image Processing”, Addison Wesley, (2002).

[4] P. Sheridan, T. Hintz and D. Alexander, "Pseudo Invariant Image Transformation on a Hexagonal Lattice," Image and Vision Computing, vol. 18, (2000), pp. 907-917. 
[5] R. M. Mersereau, "The Processing of Hexagonally Sampled Two- Dimensional Signals," Proc. of the IEEE, vol. 67, (1979), pp. 930949.

[6] F. Faille and M. Petrou, "Invariant Image Reconstruction from Irregular Samples and Hexagonal Grid Splines," Image and Vision Computing, vol. 28, (2010), pp. 1173-1183.

[7] L. Middleton and J. Sivaswamy, "Edge Detection in a Hexagonal Image Processing Framework", Image and vision computing, vol. 19, (2001), pp. 1071-1081.

[8] X. He, W. Jia, Q. Wu, N. Hur, T. Hintz and H. Wang, "Basic Transformations on Virtual Hexagonal Structure", Proceedings of the International Conference on Computer Graphics, Imaging and Visualization, (2006).

[9] T. Shigeki and M. Okutomi, "Comparison of Image Alignment on Hexagonal and Square Lattices", Proceeding of IEEE $17^{\text {th }}$ International Conference on Image Processing, (2010) September 26-29, Hong Kong.

[10] X. Ho, T. Hintz, Q. Wu, H. Wang and W. Jia, "A New Simulation of Spiral Architecture", Proceeding of International Conference on Image processing, Computer Vision and Pattern Recognition, (2006) June, Las Vegas.

[11] F. Asharindavida, N. Hundewale and S. Aljahdali, "Introduction of Pseudo Hexagonal Pixels and Virtual Hexagonal Structure", International Conference on Information and Knowledge Management of Taif University, vol. 45, (2012), Saudi Arabia.

[12] L. Middleton and J. Sivaswamy, "Hexagonal Image Processing,” A Practical Approach, London, (2005).

[13] H. Wang, M. Wang, T. Hintz, X. He and Q. Wu, "Pseudo Spiral Architecture", University of Technology, Sydney, (2007).

[14] T. Hintz, Q. Wu and X. He, "Mimic Spiral Architecture", University of Technology, Sydney, (2007).

[15] S. Veni, "Vision Based Hexagonal Image Processing," PHD Thesis, Department of Electronics and Communication Engineering, India, (2012) January.

[16] X. He, “2-D object Recognition with Spiral Architecture,” PHD Thesis, University of Technology, Sydney, (1999).

\section{Authors}

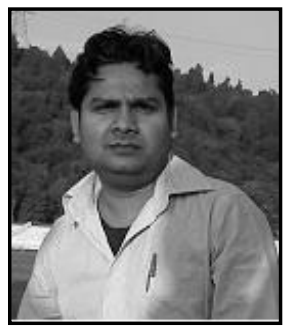

Barun Kumar, $4^{\text {th }}$ Sem, M.Tech Electronics \& Communication MMU, Mullana, Ambala, Haryana, India.

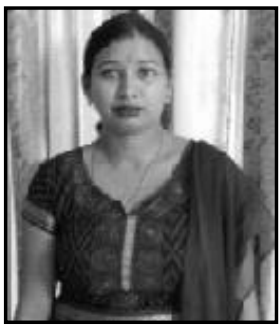

Pooja Gupta, Assistant Professor Department of Electronics \& Communication MMU, Mullana, Ambala, Haryana, India.

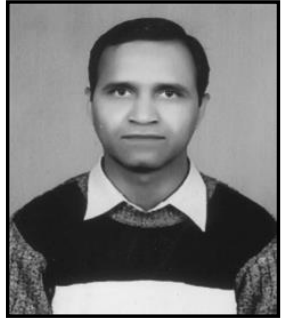

Dr. Kuldip Pahw, Professor Department of Electronics \& Communication MMU, Mullana, Ambala, Haryana, India. 